\title{
IL CARATTERE DIALETTICO DEL COGITO
}

Il famoso metodo cartesiano è senza dubbio un metodo intuitivo-deduttivo, cioè consiste nel partire da intuizioni assolutamente certe, le quali fungono da principi, e nel dedurne le conseguenze che necessariamente ne derivano. Esso è infatti il metodo della matematica, più precisamente della geometria.' Invece il modo seguito da Descartes per stabilire i principi, almeno per quanto riguarda il Cogito, è completamente diverso e può essere definito dialettico, se con questo termine si intende non l'arte scolastica della disputa, cone pensava lo stesso Descartes, bensì la dimostrazione per confutazione, praticata da Socrate, da Platone e da Aristotele nella difesa del principio di non-contraddizione.

Ciò risulta chiaramente da tre ordini di considerazioni. I) Descartes, per stabilire quali sono i principi assolutamente certi, decide anzitutto di considerare falso tutto ciò che può essere revocato in dubbio, ciod applica un procedimento di tipo negativo, consistente nel negare la verita di tutta una serie di opinioni. Ciò corrisponde alla prima operazione della dialettica anti$\mathrm{ca}$, che è quella di tentare di confutare qualunque soluzione venga proposta dal proprio interlocutore alla formulazione di un problema.

2) Il dubitare di tutto mostra l'indubitabilità della propria esistenza, ciod del Cogito, perché questo è il dubitare stesso. Ciò corrisponde alla seconda operazione della dialettica antica, che è quella di mostrare l'innegabilitả di ciò per mezzo di cui si confuta, in particolare del principio di non-contraddizione, attraverso la confutazione di ogni tentativo di negarlo. 3) Descartes ripetutamente esclude che il procedimento seguito per giungere al Cogito sia un sillogismo, cioè la deduzione di una conclusione particolare da una premessa universale, e dichiara che essa d̀ una nozione prima, di carattere particolare, oggetto di una conoscenza interiore. Ciò corrisponde alla tesi dell'indimostrabilitả dei principi e della loro accessibilită solo per via di induzione, cioè per mezzo della dialettica, sostenuta da Aristotele negli Analitici posteriori e nei Topici.

\section{Il dubbio universale}

I passi in cui Descartes propone il dubbio universale nella forma di un procedimento negativo, consistente nel negare la verità delle opinioni recepite in precedenza, sono notissimi. II primo è quello contenuto nella parte IV del Discorso sul metodo, in cui l'autore dichiara: "ritenni necessario ... rigettare come interamente falso tutto ciò in cui potessi immaginare il menomo dubbio, per vedere se, cosi facendo, alla fine, restasse qualcosa, nella mia credenza, di assolutamente indubitabile», e sottopone a questo procedimento sia le attestazioni dei sensi, sia le conclusioni dei ragionamenti, sia in generale tutti i pensieri che abbiamo quando siamo desti.?

A ciò fa eco la prima delle Meditazioni metafisiche, in cui l'autore afferma: umi applicherò seriamente e con libertà a una distruzione generale di tutte le mie antiche opinioni. E non sard necessario, per arrivare a questo, provare che esse sono tutte false ..., ma il menomo moti-

I A qutesto proposito mi permetto di rinviare al mio articolo Le origini del mntematismo modemo, "Giomale critico della filosofia italiana», 51, 1972, pp. 337-365.

2 Cfr. Cantesio, Opere, a cura di E. Garin, Bari, Lalerza, 1967, vol. I, p. 151. 
vo di dubbio che troverò basterà per farmele tutte rifiutare». ${ }^{3} \mathrm{Ma}$ anche nelle Risposte alle seconde Obiezioni, aggiunte alle Meditazioni, Descartes dichiara di non conoscere unulla di più utile per pervenire ad una ferma e sicura conoscenza delle cose che, prima di nulla stabilire, abituarsi a dubitare di tutton; 4 e nelle Risposte alle settime Obiezioni propone il celebre paragone del cesto di mele, che deve essere vuotato tutto per consentire di individuare quali sono le mele sane e separarle da quelle guaste.

«Proprio cosi -egli commenta - anche quelli che non hanno mai ben filosofato hanno diverse opinioni nel loro spirito, che hanno cominciato ad ammassarvi fin dalla loro tenera età; e ritenendo con ragione che la maggior parte non siano vere, cercano di separarle dalle altre, per paura che la loro mescolanza non le renda tutte incerte. E per non ingannarsi essi non saprebbero far meglio che respingerle tutte insieme una buona volta, né più né meno che se fossero tutte false e incerte; per poi, esaminandole per ordine le une dopo le altre, riprender solo quelle che essi riconosceranno come vere e indubitabilis. 5

Questo procedimento negativo, oltre ad essere il segno di un alteggiamento radicalmente critico, quale si conviene ad una concezione rigorosa della filosofia come edomandare tutto che $\dot{e}$ tutto domandare ${ }^{6}$ corrisponde perfettamente a quella che si potrebbe chiamare la prima delle operazioni della dialettica antica, ciò̀ la confutazione (èlenchos). Questa, come è noto, fu praticata ampiamente da Socrate, il quale, come risulta dai primi dialoghi platonici, in genere poneva ai suoi interlocutori una domanda e, una volta oltenuta la risposta, si applicava a confutarla deducendo da altre risposte ottenute dagli interlocutori una conclusione che fosse in contraddizione con essa. ${ }^{7}$ Essa fu inoltre teorizzata da Platone, il quale nella Repubblica afferma addirittura cle, per giungere alla conoscenza dell'idea del bene, è necessario "passare attraverso tutte le confutazioni (dià panton elènchon), come in battaglia, e confutare non secondo opinione, ma secondo realtàm. 8

Ma anche Aristotele, nella Metafisica, afferma che la prima operazione da compiersi per avanzare nella scienza ricercata è quella di formulare le "aporie» (aporésai), contrapponendo fra loro le soluzioni fornite dall'opinione, e sviluppare le soluzioni opposte (diaporèsai), deducendo da ciascuna di esse tutte le conseguenze che ne derivano, per vedere se si giunga a qualche contraddizione con l'una o l'altra di esse. ${ }^{9}$ E nei Topici dichiara che questo è l'uso filosofico della dialettica, perché solo dopo avere sviluppato le aporie in entrambe le direzioni (eis amplòtera diaporèsai), per vedere sc esse approdino a qualche contraddizione, si può giungere a scorgere più facilmente il vero e il falso. ${ }^{10}$ Aristotele anzi analizza dal punto di vista logico la struttura della confutazione, affermando che essa è il «sillogismo della contraddizione» (antipháseos sullogismos), cioè la deduzione di conclusioni contraddittorie rispetto a una certa tesi, I' e

3 Ivi, p. 199.

4 lvi, p. 306.

IVi, p. 633.

6 Cfr. M. Gentile, Filosofia e mmanesimo, Btescia, La Scuola, 1947.

7 Un esempio emblematico di questo procedimento si puó trovare nell'Eutifrone (7 A-8 A), dove Socrate alla doinanda "che cosa è santo?" ottiene la risposta "santo è ciò cle piace agli dèi»; dopo di che egli domanda a Eutifrone se gli déi sono uno o molti, e ne ottiene la risposta che sono molti; se sono concordi o discordi. e ne ottiene la risposta che sono discordi; allora conclude che la stessa azione ad alcuni dài piacerà e ad altri non piacerà, per cui sard santa ed insieme non santa.

8 Plat. Resp. VII, 534 B-C.

9 Aristot. Metaph. III 1,995 a 24-36.

10 Aristot. Top. 1 2, 101 a 34- b 4.

It Aristot. An. pr. Il 20,66 b 6-11. 
teorizza la contraddizione come segno di falsità nella sua celebre formulazione del principio di non-contraddizione. ${ }^{12}$

\section{L'indubitabilita del dubbio}

$\mathrm{Ma}$, come è noto, proprio dal dubbio universale Descartes ricava la sua prima certezza, ciod il principio fondamentale di tutta la sua filosofia, vale a dire il Cogilo. Se, infatti, si mette il discussione lo stesso dubitare, non lo si nega, ma lo si riafferma, perche il mettere in discussione 3 una forma di dubbio, dunque il dubbio risulta indubitabile, e il dubbio non è altro che il Cogito. Ciò risulta anzitutto dal celebre passo, sempre nella parte IV del Discorso sul metodo, dove Descartes afferma: «ma, subito dopo [aver dubitato], m'accorsi che, mentre volevo in tal modo pensare falsa ogni cosa, bisognava necessariamente che io, clie la pensavo, fossi pur qualcosa. Per cui, dalo che questa verità: lo penso, dunque sono, ̀̀ cosi ferma e certa cle non avrebbero potuto scuoterla neanche le più stravaganti supposizioni degli scettici, giudicaj di poterla accogliere senza esitazione come il principio primo della mia filosofiam. ${ }^{13}$

La stessa certezza è raggiunta, nella seconda delle Meditazioni metafisiche, attraverso l'ipotesi del genio ingannatore: «io esistevo senza dubbio, se mi sono convinto di qualcosa, o se solamente ho pensato qualcosa. Ma vi è un non so quale ingannatore potentissimo e astutissimo, che impiega ogni suo sforzo nell'ingannarmi sempre. Non v'è dubbio che io esisto, s'egli mi inganna; e m'inganni fin che vorrà, egli non saprà mai fare che io non sia nulla, fino a che penserò di essere qualcosan. ${ }^{14}$ Fino a questo punto può sembrare che la ccrtezza in questione sia solo quella della propria esistenza: in realtà essa ì anzitutto quella del pensiero, di un pensiero che si esercita nella forma del dubitare, un dubitare dunque che si rivela indubitabile.

Ciò risulta chiaro da altri due passi, l'uno contenuto nelle Risposte alle settime Obiezioni, che dice: «dopo aver considerato che non si poteva dubitare che la sostanza che dubita cosi di tutto, o che pensa, non esistesse mentre dubita, mi sono servito di questo come d'una terra ferma, sulla quale poggiare le fondamenta della mia filosofiam;15 l'altro contenuto nei Principia philosophiae, che suona: «non sapremmo supporre in egual modo [a quello con cui dubitiamo di tutto] che noi non esistiamo mentre dubitiamo della verità di tutte queste coses. 16 Qui la certezza del pensare, e quindi dell'esistere, a data dal fatto di dubitare: è il dubbio dunque, prima ancora del pensicro e dell'esistenza, cle è indubitabile.

Anche di questa operazione si può trovare un analogo nella dialettica antica, precisamente nel momento in cui lo strumento per mezzo del quale si confutano le opinioni, cioe il principio di non-contraddizione, risulta esso stesso inconfutabile, ma lo risulta proprio altraverso la confutazione del tentativo di negarlo. Il referente, in questo caso, non può che essere Aristotele, il quale, dopo avere alfermato cle il principio di non-contraddizione è indimostrabile, perclié è la condizione di tutte le dimostrazioni, aggiunge che esso può essere dimostrato solo per mezzo di una dimostrazione (elenktikos), a condizione cle qualcuno lo neghi dicendo qualche cosa di determinato. Nell'atto di dire questo, infatti, il negatore implicitamente escluderì l'op-

12 Aristot. Metaph. IV 3, 1005 b 19-22. Per un'analisi della dialettica antica devo rinviare al mio libro Contraddizione e dialeatica negli antichi e nei moderni, Palermo. L'epos, 1987.

13 Cartesio. Opere cit., p. 151.

14 Jvi, p. 206.

is Jvi, p. 683 (corsivo mio).

16 Ivi, vol. II, p. 28 (corsivo mio). 
posto di quello che dice, e quindi affemerà il principio di non-contraddizione, cadendo in tal modo in una petizione di principio e risultando quindi confutato. 17

\section{Il Cogito non risulta dal metodo matematico}

Infine una terza analogia tra il procedimento impiegato da Descartes per stabilire il Cogito, cioè il principio di tutta la sua filosofia, risulta dall'affermazione, fatta più volte dall'autore, che tale procedimento non è un sillogismo, cioè non è la deduzione di una premessa particolare da una conclusione universale, il che significa che esso non puó essere dimostrato col procedimento deduttivo proprio del metodo matematico. Tale affermazione si trova anzitutto nelle Risposte alle seconde Obiezioni, dove si dice: «quando ci accorgiamo di essere delle cose pensanti, $\mathrm{e}$ questa una nozione prima, che non è tratta da nessun sillogismo; e quando qualcuno dice: lo penso, dunque io sono, o csisto, non deduce la sua esistenza dal suo pensiero per forza di sillogismo, ma come cosa conosciuta per sé la vede come una semplice intuizione della menten.18

Essa poi ritorna nelle Risposte alle quinte Obiczioni, dove Descartes risponde a Gassendi, il quale pretendeva che il cogito fosse dedotto dalla premessa universale "colui che pensa, è, nel modo seguente: "l'errore che qui è il più considerevole è che questo autore suppone clıe la conoscenza delle proposizioni particolari debba essere sempre dedotta dalle universali, secondo l'ordine dei sillogismi della dialettica; nel che mostra di sapere assai poco in che modo la verità si deve ricercare; poiché è certo che, per trovarla, si deve sempre cominciare dalle nozioni particolari, per venire in appresso alle generalin. 19

La stessa tesi viene esposta in forma più ampia nel Colloquio con Burnan, dove, commentando il primo dei due passi qui riportati, si dice: «prima di questa conclusione: penso dunque sono, si può conoscere la premessa maggiore: tutto ciò che pensa $\dot{e}$, perchá realmente essa precede la mia conclusione e ne costituisce il fondamento. Perciò l'autore dice nei Principi che essa precede in quanto, implicitamente, d sempre presupposta e precede; ma non sempre conosco la sua anteriorità in forma espressa ed esplicita, e conosco per prima la mia conclusione, perché bado soltanto a ciò che sperimento in me stesso, come penso dunque sono, mentre non faccio altrettanta attenzione alla nozione generica tutto ció che pensa è». ${ }^{20}$

Anche Aristotele, negli Analifici posteriori, dichiara che i principi delle scienze non possono essere stabiliti per via dimostrativa, cioc̀ mediante un sillogisno scientifico, ma devono essere raggiunti per mezzo dell'induzione (epagogè), cioè di un procedimento che muove da casi particolari, conosciuti per mezzo della percezione, per salire verso conclusioni di carattere generale, cios universale. ${ }^{21}$ E nei Topici afferma che la via che conduce ai princìpi è posseduta solo dalla dialettica, perché questa è uatta ad esaminare» (exetastiké), cioè a mettere alla prova, a confutare. 22

Evidentemente Descartes aveva una concezione diversa della dialettica, da lui considerata una logica puramente formale e quindi vuota di conoscenza. Ma il sillogismo con cui alcuni presumevano di dimostrare it cogito non era dialettico, nel senso antico del termine, bensi scientifico, cioè riconducibile al metodo della scienza dimostrativa, cle anche per Aristotele era rappresentata soprattutto dalla matematica. Il fatto che Descartes escluda la possibilità di dedu-

\footnotetext{
17 Aristot. Metoph. IV 4, 1005 b 35-1006 a 28

18 Cartesio, Opere cit., pp. 314-315.

19 Ivi, p. 554.

20 Ivi, vol. Il, pp. 664.665.

21 Aristot. An. post. II 19.

22 Aristot. Top. 12 cit.
} 
tre il cogito mediante un simile sillogismo, significa che egli intende sottrarre il principio primo della sua metafisica al dominio del metodo matematico, riconoscendogli un carattere non molto diverso da quello che era riconosciuto ai principi nell'ambito della dialettica antica. ${ }^{23}$

23 Ho accennato alle implicazioni che questo fatto ha per l'intera filosofia cartesiana nelf'intervento su Descartes: un problema storiografico, in AA. VV., Cartesiana, Lecce. Congedo, 1992, pp. 57-61. 